\title{
FAUNA DE COLEOPTERA COLETADA COM ARMADILHAS LUMINOSAS EM PLANTIO DE Eucalyptus grandis EM SANTA BÁRBARA, MINAS GERAIS ${ }^{1}$
}

\author{
Fernando Azevedo de Freitas², Teresinha Vinha Zanuncio ${ }^{3}$, Mabio Chrisley Lacerda² e José Cola Zanuncio ${ }^{3}$
}

\begin{abstract}
RESUMO - O objetivo deste trabalho foi estudar a flutuação populacional de espécies da ordem Coleoptera (Insecta) em plantios de Eucalyptus grandis na região de Santa Bárbara, Minas Gerais. Os coleópteros foram coletados por meio de armadilhas luminosas, acionadas durante uma noite, a cada 15 dias, de julho de 1993 a junho de 1994. Foram coletados 5.641 indivíduos da ordem Coleoptera, sendo 866 e 4.775 identificados em nível de gênero e, ou, espécie e família, respectivamente. A família com maior número de indivíduos foi Carabidae, com 74,55\%, seguida por Scarabaeidae e Elateridae, com 10,12 e 3,98\% dos indivíduos coletados, respectivamente. As espécies mais coletadas foram Isonychus albicinctus (Scarabaeidae), Colaspis jolivetti (Chrysomelidae) e Cyclocephala laminata (Scarabaeidae), com 472, 317 e 16 indivíduos, correspondendo a 54,50, 36,61 e 1,85\% dos indivíduos identificados por espécie, respectivamente. A região de Santa Bárbara apresenta fauna diversificada de espécies da ordem Coleoptera, mas com baixas possibilidades de surtos. As maiores densidades populacionais dos coleópteros ocorreram em novembro e dezembro, época em que se deve realizar o monitoramento desses insetos.
\end{abstract}

Palavras-chave: Coleoptera, Eucalyptus grandis e flutuação populacional.

\section{FAUNA OF COLEOPTERA COLLECTED WITH LIGHT TRAPS IN A Eucalyptus grandis PLANTATION IN SANTA BÁRBARA, MINAS GERAIS, BRAZIL}

\begin{abstract}
The objective of this research was to study population fluctuation of Coleoptera species in the county of Santa Bárbara, Minas Gerais, Brazil in a Eucalyptus grandis plantation. Coleoptera individuals were collected with five light traps during one night every fifteen days from July 1993 to June 1994. A total of 5,641 individuals of this order was collected, being 866 and 4,775 identified at genus and/or species and family levels, respectively. The family with the highest number of individuals was Carabidae with 74.55\%, followed by Scarabaeidae and Elateridae with $10.12 \%$ and $3.98 \%$ of individuals of this group, respectively. Most species collected were Isonychus albicinctus (Scarabaeidae), followed by Colaspis jolivetti (Chrysomelidae) and Cyclocephala laminata (Scarabaeidae) with 412, 317 and 16 individuals which represented 54.50, 36.61 and $1.85 \%$ of individuals identified per species, respectively. The region of Santa Bárbara presents a diversified fauna of Coleoptera, but with low outbreak possibilities. Population peaks of Coleoptera species ocurred in November and December, when monitoring of these insects is recommended.
\end{abstract}

Key words: Coleoptera, Eucalyptus grandis, population fluctuation.

\section{INTRODUÇÃO}

O reflorestamento é um dos setores com maior crescimento no Brasil, sendo o Estado de Minas Gerais o líder nessa atividade, com a maioria das áreas com espécies do gênero Eucalyptus (Zanuncio et al., 1992a). $\mathrm{O}$ aumento da área reflorestada com essências exóticas tem levado ao aumento de problemas com insetos-praga (Santos et al., 1982; Zanuncio et al., 1994a), em razão das modificações ambientais, nas monoculturas, que

1 Recebido para publicação em 19.4.2001.

Aceito para publicação em 20.6.2002.

2 Bolsistas de Aperfeiçoamento da FAPEMIG, Universidade Federal de Viçosa - UFV, 36571-000 Viçosa-MG. ${ }^{3}$ Professores do Departamento de Biologia Animal/BIOAGRO, UFV, <zanuncio@mail.ufv.br>. 
levam ao empobrecimento geral da fauna. Por outro lado, a menor competição favorece a proliferação e afeta a dinâmica populacional de insetos-praga, pela maior disponibilidade de alimento e menor diversidade e número de indivíduos de inimigos naturais (Mezzomo et al., 1998; Pereira et al., 1994; Zanuncio et al., 1995).

O fato de os plantios florestais serem, normalmente, constituídos por monoculturas em vastas extensões de terra e cultivados por longos períodos tem favorecido as espécies-praga, principalmente as do grupo das formigascortadeiras, dos lepidópteros-desfolhadores e dos coleópteros, os quais constituem os maiores problemas para a eucaliptocultura nacional (Anjos et al., 1986, Zanuncio et al., 1991, 1992a, 1992b, 1993a, 1993b, 1994b). Embora as espécies dos primeiros grupos sejam mais bem estudadas, os coleópteros associados a plantios de eucalipto são menos conhecidos, apesar de algumas famílias desse grupo terem espécies-praga para a eucaliptocultura (Zanuncio et al., 1993a). Neste grupo estão incluídas as espécies do gênero Xyleborus (Scolytidae), denominado besouro - ambrósia, e Neoclytus sp. (Cerambycidae), que é broqueadora de madeira. Outros insetos da ordem Coleoptera, como Costalimaita ferruginea (Chrysomelidae) e Bolax flavolineatus (Scarabaeidae), ocorrem no início das chuvas e têm causado sérios danos em plantios jovens de eucalipto (Zanuncio et al., 1993a). Além disto, a ordem Coleoptera também apresenta espécies que atacam sementes de essências nativas, especialmente das famílias Bruchidae, Anthribidae e Cerambycidae (Santos et al., 1991, 1997). A primeira família apresenta, também, espécies de importância agrícola, cujo ataque afeta a germinação e a qualidade de sementes, principalmente quando armazenadas (Carvalho \& Figueira, 1999).

Portanto, o objetivo deste trabalho foi estudar a flutuação populacional de espécies da ordem Coleoptera em plantio de Eucalyptus grandis W. Hill ex Maiden, no município de Santa Bárbara, Minas Gerais, de julho de 1993 a junho de 1994, permitindo caracterizar a sua fauna, distribuição temporal e densidade populacional e possibilitando o manejo integrado correto das espéciespraga da ordem Coleoptera na região.

\section{MATERIAL E MÉTODOS}

Este estudo foi realizado em um plantio de Eucalyptus grandis localizado na região de Santa Bárbara, Minas Gerais, de julho de 1993 a junho de 1994. Os coleópteros foram coletados com cinco armadilhas luminosas do tipo Intral AL - 012, instaladas $2 \mathrm{~m}$ de altura do solo, providas de luz negra e acionadas, quinzenalmente, durante uma noite, por bateria de 12 volts (Zanuncio et al., 1993a; Pereira et al., 2001). Um saco plástico, contendo em seu interior tiras de papel e um vidro com acetato de etila, foi acoplado ao funil de cada armadilha, para diminuir os danos morfológicos aos exemplares coletados (Ferreira \& Martins, 1982).

Os insetos coletados foram acondicionados em mantas entomológicas, etiquetadas com o local e a data da coleta, e enviados ao Insetário do Departamento de Biologia Animal da Universidade Federal de Viçosa (UFV), Minas Gerais, onde foram contados e catalogados e aqueles em boas condições, montados. A identificação foi baseada nas comparações com exemplares depositados nas coleções entomológicas da UFV, bem como na literatura. Em seguida, foram divididos em dois grupos, identificados em nível de gênero e, ou, espécie e em nível de família, sendo os dados desses insetos transformados em $\log (x+1)$, para confecção do gráfico de sua flutuação populacional.

\section{RESULTADOS E DISCUSSÃO}

De julho de 1993 a junho de 1994 foram coletados 5.641 coleópteros, sendo 866 identificados em nível de gênero e, ou, espécie e 4.775 em nível de família, representando 15,35 e $84,65 \%$ do total de coleópteros coletados, respectivamente (Quadro 1).

Quadro 1 - Número e porcentagem de indivíduos da ordem Coleoptera coletados em um plantio de Eucalyptus grandis, na região de Santa Bárbara, Minas Gerais. Julho de 1993 a junho de 1994

Table 1 - Number and percentage of individuals of the Coleoptera order collected in a Eucalyptus grandis plantation in the county of Santa Bárbara, Minas Gerais, Brazil. July 1993 to June 1994

\begin{tabular}{|c|c|c|c|c|}
\hline \multirow{2}{*}{ Grupo } & \multicolumn{2}{|c|}{ Número de Espécies } & \multicolumn{2}{|c|}{ Número de Indivíduos } \\
\hline & Total & $\%$ & Total & $\%$ \\
\hline Identificados por gênero e, ou, espécie & 19 & 7,98 & 866 & 15,35 \\
\hline Identificados por família & 219 & 92,02 & 4.775 & 84,65 \\
\hline Total & 238 & 100,00 & 5.641 & 100,00 \\
\hline
\end{tabular}


Os picos populacionais de espécies de Coleoptera foram registrados em novembro e dezembro, com $978 \mathrm{e}$ 3.358 indivíduos, respectivamente, o que mostra que estes insetos apresentam populações mais elevadas na época mais quente e úmida do ano. Já os menores números de indivíduos foram observados nos meses de junho, julho e agosto, com zero, cinco e quatro indivíduos, respectivamente (Quadros 2 e 3, Figura 1), quando são registradas as menores temperaturas do ano e a precipitação pluvial é nula ou muito baixa. Esses dados são concordantes com os de Zanuncio et al. (1993a), que coletaram maior número de indivíduos da ordem Coleoptera em novembro e dezembro, correspondendo à época mais quente e úmida do ano, com armadilhas etanólicas e luminosas em plantios de eucalipto. Já Pinto et al. (2000) coletaram maior número de coleópteros, com armadilhas luminosas, também na época mais quente e úmida do ano, com picos populacionais em setembro, outubro e março. No entanto, Morales et al. (1999, 2000), em levantamento de coleópteros da família Scolytidae com armadilhas etanólicas, mostraram que as principais espécies desta família, em plantios de E. grandis no município de Antonio Dias, Minas Gerais, apresentaram maiores níveis de abundância na época mais seca do ano, para 15 das 16 espécies coletadas neste período. Tal fato mostra que o conhecimento dos fatores que afetam a flutuação populacional de insetos é importante para prever a tendência da mesma (Morales et al., 2000) e que se deve utilizar um tipo ou outro de armadilha, de acordo com o grupo de espécies da ordem Coleoptera que se quer estudar.

Quadro 2 - Número de indivíduos da ordem Coleoptera, coletados com armadilhas luminosas e identificados em nível de gênero e, ou, espécie em plantios de Eucalyptus grandis, na região de Santa Bárbara, Minas Gerais. Julho de 1993 a junho de 1994

Table 2 - Number of individuals of the Coleoptera order, collected with light traps and identified by genus and/or species in a Eucalyptus grandis plantation in the county of Santa Bárbara, Minas Gerais, Brazil. July 1993 to June 1994

\begin{tabular}{|c|c|c|c|c|c|c|c|c|c|c|c|c|c|c|}
\hline \multirow{2}{*}{ Família/Gênero e, ou, Espécie } & \multicolumn{6}{|c|}{1993} & \multicolumn{6}{|c|}{1994} & \multirow{2}{*}{ Total } & \multirow{2}{*}{$\%$} \\
\hline & Jul. & Ago. & Set. & Out. & Nov. & Dez. & Jan. & Fev. & Mar. & Abr. & Maio & Jun. & & \\
\hline Carabidae & & & & & & & & & & & & & & \\
\hline Galerita lacordairei & 0 & 0 & 1 & 0 & 0 & 1 & 0 & 0 & 0 & 0 & 0 & 0 & 2 & 0,23 \\
\hline Pleuracanthus genus & 0 & 0 & 1 & 0 & 1 & 1 & 0 & 0 & 0 & 0 & 0 & 0 & 3 & 0,35 \\
\hline Chrysomelidae & & & & & & & & & & & & & & \\
\hline Chalcophana viridipennis & 0 & 0 & 0 & 0 & 1 & 0 & 0 & 1 & 0 & 0 & 0 & 0 & 2 & 0,23 \\
\hline Colaspis jolivetti & 0 & 0 & 0 & 0 & 302 & 15 & 0 & 0 & 0 & 0 & 0 & 0 & 317 & 36,61 \\
\hline Costalimaita sp. & 0 & 0 & 0 & 0 & 4 & 4 & 0 & 0 & 0 & 0 & 0 & 0 & 8 & 0,92 \\
\hline Diphaulaca volkameriae & 0 & 0 & 0 & 1 & 0 & 0 & 0 & 0 & 0 & 0 & 0 & 0 & 1 & 0,12 \\
\hline Maecolaspis laeta & 0 & 0 & 0 & 0 & 1 & 0 & 0 & 0 & 0 & 0 & 0 & 0 & 1 & 0,12 \\
\hline Cicindelidae & & & & & & & & & & & & & & \\
\hline Oxychila tristis & 0 & 0 & 0 & 0 & 1 & 0 & 0 & 0 & 0 & 0 & 0 & 0 & 1 & 0,12 \\
\hline Curculionidae & & & & & & & & & & & & & & \\
\hline Heilipodus sp. & 1 & 0 & 0 & 0 & 1 & 1 & 0 & 0 & 1 & 1 & 0 & 0 & 5 & 0,58 \\
\hline Heilipus lactarius & 0 & 0 & 1 & 0 & 0 & 0 & 0 & 0 & 0 & 0 & 0 & 0 & 1 & 0,12 \\
\hline Rhinostomus barbirostris & 0 & 0 & 0 & 0 & 1 & 0 & 0 & 0 & 0 & 0 & 0 & 0 & 1 & 0,12 \\
\hline Scarabaeidae & & & & & & & & & & & & & & \\
\hline Belloceras sp. & 0 & 0 & 0 & 7 & 0 & 7 & 0 & 0 & 1 & 0 & 0 & 0 & 15 & 1,73 \\
\hline Bolax zubkoffi & 0 & 0 & 0 & 1 & 0 & 0 & 0 & 0 & 0 & 0 & 0 & 0 & 1 & 0,12 \\
\hline Cyclocephala laminata & 0 & 0 & 0 & 12 & 1 & 2 & 0 & 0 & 1 & 0 & 0 & 0 & 16 & 1,85 \\
\hline Geniates laevis & 0 & 0 & 0 & 3 & 0 & 0 & 0 & 0 & 1 & 0 & 0 & 0 & 4 & 0,46 \\
\hline Isonychus albicinctus & 0 & 0 & 432 & 33 & 2 & 4 & 0 & 0 & 1 & 0 & 0 & 0 & 472 & 54,50 \\
\hline Pelidnota aeruginosa & 0 & 0 & 0 & 0 & 1 & 0 & 0 & 0 & 0 & 0 & 0 & 0 & 1 & 0,12 \\
\hline $\begin{array}{l}\text { Silphidae } \\
\text { Oxelytrum discicolle }\end{array}$ & 0 & 0 & 1 & 0 & 0 & 5 & 3 & 0 & 0 & 0 & 1 & 0 & 10 & 1,15 \\
\hline $\begin{array}{l}\text { Scolytidae } \\
\text { Xyleborus affinis }\end{array}$ & 0 & 0 & 0 & 0 & 0 & 0 & 0 & 0 & 5 & 0 & 0 & 0 & 5 & 0,58 \\
\hline $\begin{array}{r}\text { Total } \\
\end{array}$ & 1 & 0 & 436 & 57 & 316 & 40 & 3 & 1 & 10 & 1 & 1 & 0 & 866 & 100,00 \\
\hline
\end{tabular}


Quadro 3 - Número de indivíduos da ordem Coleoptera identificados em nível de família coletados com armadilhas luminosas em plantios de Eucalyptus grandis, na região de Santa Bárbara, Minas Gerais. Julho de 1993 a junho de 1994

Table 3 - Number of individuals of the Coleoptera order identified per family collected with light traps in a Eucalyptus grandis plantation in the county of Santa Bárbara, Minas Gerais, Brazil. July 1993 to June 1994

\begin{tabular}{|c|c|c|c|c|c|c|c|c|c|c|c|c|c|c|}
\hline \multirow{2}{*}{ Família } & \multicolumn{6}{|c|}{1993} & \multicolumn{6}{|c|}{1994} & \multirow{2}{*}{ Total } & \multirow{2}{*}{$\%$} \\
\hline & Jul. & Ago. & Set. & Out. & Nov. & Dez. & Jan. & Fev. & Mar. & Abr. & Maio & Jun. & & \\
\hline Anthribidae & 0 & 0 & 0 & 0 & 0 & 1 & 0 & 0 & 0 & 0 & 0 & 0 & 1 & 0,02 \\
\hline Brenthidae & 0 & 0 & 0 & 0 & 1 & 0 & 0 & 0 & 0 & 0 & 0 & 0 & 1 & 0,02 \\
\hline Bruchidae & 0 & 0 & 0 & 0 & 0 & 4 & 0 & 0 & 0 & 0 & 0 & 0 & 4 & 0,08 \\
\hline Carabidae & 2 & 0 & 72 & 8 & 178 & 3.039 & 22 & 76 & 91 & 32 & 40 & 0 & 3.560 & 74,55 \\
\hline Cerambycidae & 0 & 0 & 0 & 6 & 1 & 4 & 2 & 1 & 6 & 1 & 1 & 0 & 22 & 0,46 \\
\hline Chrysomelidae & 0 & 0 & 0 & 0 & 101 & 18 & 2 & 2 & 0 & 0 & 0 & 0 & 123 & 2,58 \\
\hline Cicindelidae & 0 & 0 & 0 & 0 & 1 & 0 & 0 & 0 & 0 & 0 & 0 & 0 & 1 & 0,02 \\
\hline Coccinelidae & 0 & 0 & 0 & 1 & 1 & 5 & 0 & 8 & 9 & 2 & 0 & 0 & 26 & 0,54 \\
\hline Curculionidae & 0 & 0 & 5 & 0 & 10 & 14 & 6 & 37 & 9 & 0 & 11 & 0 & 92 & 1,93 \\
\hline Dermestidae & 0 & 0 & 1 & 0 & 0 & 22 & 0 & 0 & 0 & 0 & 0 & 0 & 23 & 0,48 \\
\hline Dytiscidae & 0 & 0 & 0 & 0 & 1 & 2 & 0 & 0 & 0 & 0 & 0 & 0 & 3 & 0,06 \\
\hline Elateridae & 0 & 0 & 1 & 4 & 66 & 91 & 6 & 8 & 8 & 6 & 0 & 0 & 190 & 3,98 \\
\hline Erotylidae & 0 & 0 & 2 & 0 & 4 & 5 & 0 & 2 & 5 & 1 & 0 & 0 & 19 & 0,40 \\
\hline Hidrophylidae & 0 & 0 & 0 & 0 & 0 & 1 & 0 & 0 & 0 & 0 & 0 & 0 & 1 & 0,02 \\
\hline Lampyridae & 0 & 0 & 0 & 19 & 6 & 1 & 0 & 0 & 0 & 0 & 0 & 0 & 26 & 0,54 \\
\hline Lycidae & 0 & 0 & 0 & 0 & 0 & 0 & 0 & 3 & 2 & 1 & 0 & 0 & 6 & 0,13 \\
\hline Meloidae & 0 & 0 & 0 & 0 & 2 & 1 & 0 & 0 & 1 & 0 & 0 & 0 & 4 & 0,08 \\
\hline Nitidulidae & 0 & 0 & 11 & 0 & 8 & 11 & 4 & 2 & 2 & 2 & 0 & 0 & 40 & 0,84 \\
\hline Platypodidae & 0 & 0 & 0 & 0 & 0 & 0 & 0 & 0 & 1 & 0 & 0 & 0 & 1 & 0,02 \\
\hline Scarabaeidae & 2 & 4 & 11 & 115 & 214 & 87 & 21 & 5 & 14 & 3 & 7 & 0 & 483 & 10,12 \\
\hline Scolytidae & 0 & 0 & 0 & 0 & 65 & 6 & 2 & 1 & 10 & 0 & 0 & 0 & 84 & 1,76 \\
\hline Staphylinidae & 0 & 0 & 0 & 0 & 0 & 2 & 1 & 3 & 19 & 25 & 3 & 0 & 53 & 1,11 \\
\hline Tenebrionidae & 0 & 0 & 0 & 0 & 3 & 4 & 0 & 0 & 4 & 0 & 1 & 0 & 12 & 0,25 \\
\hline Total & 4 & 4 & 103 & 153 & 662 & 3318 & 66 & 148 & 181 & 73 & 63 & 0 & 4.775 & 100,00 \\
\hline
\end{tabular}

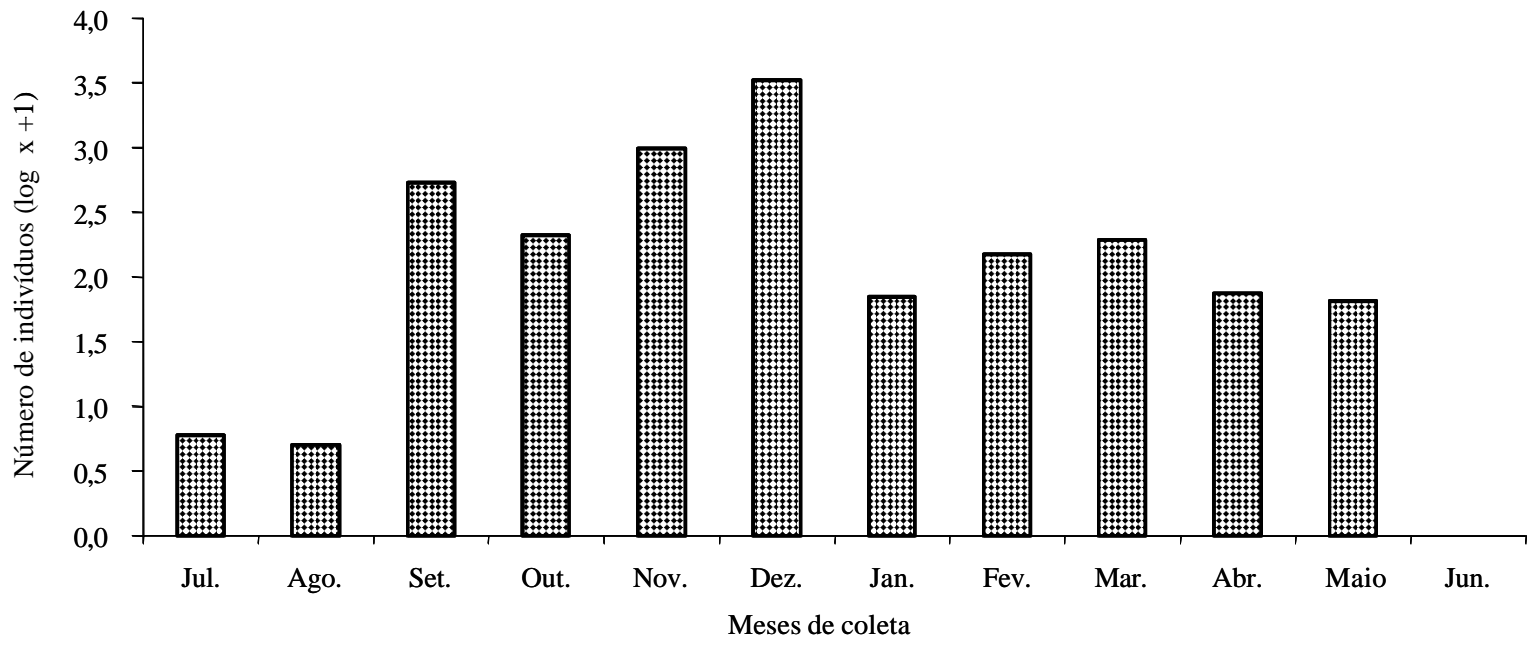

Figura 1 - Número total $(\log \mathrm{x}+1)$ de indivíduos da ordem Coleoptera coletados com armadilhas luminosas em plantios de Eucalyptus grandis, na região de Santa Bárbara, Minas Gerais, Brasil. Julho de 1993 a junho de 1994.

Figure 1 - Total number $(\log x+1)$ of individuals of the Coleoptera order collected with light traps in a Eucalyptus grandis plantation in the county of Santa Bárbara, Minas Gerais, Brazil. July 1993 to June 1994. 
Dos coleópteros identificados em nível de espécie, as três mais coletadas foram Isonychus albicinctus (Scarabaeidae), Colaspis jolivetti (Chrysomelidae) e Cyclocephala laminata (Scarabaeidae), com 472, 317 e 16 indivíduos cada (Quadro 2), representando 54,50, 36,61 e $1,85 \%$, respectivamente, o que corresponde a $92,96 \%$ do total de indivíduos identificados desse grupo (Quadro 2). Este resultado mostra a maior adaptabilidade dessas espécies aos plantios de eucalipto na região de Santa Bárbara, embora elas não tenham sido ainda relatadas e reconhecidas como insetos-praga de eucalipto. Essas espécies apresentaram picos populacionais de setembro a dezembro, com I. albicinctus no mês de setembro, $C$. jolivetti em novembro e $C$. laminata em outubro, com a primeira representando $49,42 \%$ dos indivíduos da família Scarabaeidae. Além disso, picos populacionais dessas espécies foram registrados em meses de início da época chuvosa, com poucos indivíduos sendo coletados nos demais meses do ano (Quadro 2). Este comportamento populacional é semelhante ao de outras espécies de Coleoptera importantes para a eucaliptocultura, que apresentam altas populações no início das chuvas como Costalimaita ferruginea (Chrysomelidae) e Bolax flavolineatus (Scarabaeidae) (Zanuncio et al., 1993a). Estes coleópteros pertencem às famílias das espécies mais coletadas no presente trabalho e causam danos às folhas de eucalipto, com perfurações e rendilhamentos das mesmas. Por isto, C. ferruginea e B. flavolineatus têm sido controlados com produtos químicos em determinadas situações (Anjos et al., 1986). Como I. albicinctus, $C$. jolivetti e C. laminata podem vir a causar danos ao eucalipto, é necessário o desenvolvimento de estudos sobre diferentes aspectos dessas espécies, incluindo nível de dano, biologia e métodos de controle.

Foram identificados indivíduos de 23 famílias de Coleoptera, com destaque para Carabidae, Scarabaeidae e Elateridae, que apresentaram 3.560, 483 e 190 indivíduos, o que corresponde a $74,55,10,12$ e $3,98 \%$ dos indivíduos desse grupo, respectivamente (Quadro 3).

A família Carabidae representou $63,20 \%$ dos coleópteros coletados, com pico populacional de 3.039 indivíduos em dezembro (Quadro 3). As larvas e os adultos desta família são importantes em plantios de eucalipto, por serem predadores de Lepidoptera desfolhadores (Gallo et al., 1988, Zanuncio et al., 1993a). Pinto et al. (2000) relataram que em plantios de Eucalyptus urophylla no município de Três Marias, Minas Gerais, essa família apresentou maior número de indivíduos em coletas com armadilhas luminosas. No entanto, foram registrados picos populacionais em setembro em vez de dezembro, seguida pelas famílias Aphodiidae e Melolonthidae (Pinto et al., 2000).

A família Scarabaeidae, com pico populacional em novembro com 214 indivíduos (Quadro 3), apresenta destaque por ter o besouro-pardo (B. flavolineatus) como praga importante de eucalipto no Brasil (Anjos et al., 1986). Outras espécies dessa família, como Anaplognathus spp., conhecidas como "besouros-de-natal", são pragas importantes no litoral e na costa leste da Austrália, onde seus adultos se alimentam de folhas de plantas jovens de eucalipto, nas quais podem causar danos severos, enquanto suas larvas se desenvolvem no solo, alimentando-se de matéria orgânica (Ohmart \& Edwards, 1991).

A família Elateridae, com pico populacional em dezembro com 91 indivíduos (Quadro 3), apresenta espécies importantes para a eucaliptocultura, pois são predadores de pragas dessa cultura, durante sua fase jovem ou adulta (Zanuncio et al., 1993a).

As famílias Chrysomelidae e Curculionidae apresentaram 123 e 92 indivíduos, o que representou 2,58 e $1,93 \%$, respectivamente, dos indivíduos identificados em nível de família (Quadro 3). Estas famílias apresentam espécies-praga para a eucaliptocultura como $C$. ferruginea, Sternocolaspis quatuordecimcostata (Chrysomelidae), Gonipterus gibberus e Gonipterus scutellatus (Curculionidae), que podem desfolhar plantas de eucalipto, com perfurações e rendilhamentos das folhas. Outras espécies de Curculionidae cortam os ponteiros da zona de crescimento apical das plantas, consumindo inicialmente as folhas e depois o broto vegetativo do ramo, causando danos ao tecido meristemático, o que pode comprometer o crescimento normal dessas plantas (Pedrosa-Macedo, 1993). Espécies da família Chrysomelidae podem ser importantes como insetos herbívoros em plantios de eucalipto, particularmente em árvores jovens. Chrysophtharta bimaculata (Chrysomelidae) é considerado um dos insetos-praga mais importantes em florestas da Tasmânia e Austrália, onde seus adultos e larvas se alimentam de árvores jovens de eucalipto (Ohmart \& Edwards, 1991; Clarke et al., 1997). Essa espécie pode, também, desfolhar plantas de outras espécies de importância comercial (Ohmart \& Edwards, 1991).

A família Scolytidae teve apenas 84 indivíduos coletados (Quadro 3), porém suas espécies são mais

R. Árvore, Viçosa-MG, v.26, n. 4, p.505-511, 2002 
atraídas por armadilhas etanólicas (Ferraz et al., 1999 , Zanuncio et al., 1993a). Mezzomo et al. (1998) relataram que as famílias Scolytidae e Bostrichidae tiveram maior número de indivíduos coletados com armadilhas etanólicas em povoamentos de eucalipto nos municípios de Paineiras e Paraopeba, Minas Gerais. Carvalho (1984) coletou maior número de indivíduos das famílias Scolytidae e Cerambycidae em plantios de Eucalyptus urophylla e Eucalyptus saligna no município de Salto Alto, Estado de São Paulo, com armadilhas luminosas modificadas, tendo também álcool etílico como atraente.

Os levantamentos populacionais mostram a densidade populacional das diferentes espécies e permitem caracterizar as comunidades estudadas por meio dos índices faunísticos. No entanto, a análise dessas flutuações populacionais deve considerar, também, suas tendências, seus ciclos, sua sazonalidade e sua abundância relativa ao longo do tempo (Morales et al., 1999). A região de Santa Bárbara apresenta fauna diversificada de coleópteros, com 238 espécies coletadas, o que foi maior que o encontrado por Pinto et al. (2000) e Zanuncio et al. (1993a) para outras regiões. A presença de elevado número de indivíduos da família Carabidae indica que espécies dessa família podem contribuir para a redução das possibilidades de surtos de pragas nessa região. Além disto, o monitoramento de espécies da ordem Coleoptera deve ser realizado nos meses mais quentes e úmidos do ano, com armadilhas etanólicas e luminosas, para obter uma melhor análise faunística de espécies dessa ordem na região.

\section{CONCLUSÃO}

A região de Santa Bárbara, Minas Gerais, apresenta boa diversidade de espécies da ordem Coleoptera e o seu monitoramento com armadilhas etanólicas e luminosas deve ser direcionado para as épocas mais quentes e úmidas do ano, quando ocorrem os picos populacionais da maioria das espécies de Coleoptera coletadas. Devido ao elevado número de indivíduos da família Carabidae, talvez estes possam contribuir para a redução de surtos de pragas na região.

\section{AGRADECIMENTO}

À Coordenação de Aperfeiçoamento de Pessoal de Nível Superior (CAPES), ao Conselho Nacional de Desenvolvimento Científico e Tecnológico $(\mathrm{CNPq})$ e à
Fundação de Amparo à Pesquisa do Estado de Minas Gerais (FAPEMIG). À Celulose Nipo-Brasileira S.A. (CENIBRA), pelo desenvolvimento dessa pesquisa em sua área.

\section{REFERÊNCIAS BIBLIOGRÁFICAS}

ANJOS, N.; SANTOS, G. P.; ZANUNCIO, J. C. Pragas do eucalipto e seu controle. Informe Agropecuário, v. 12, n. 141, p. 50-58, 1986.

CARVALHO, A. O. R. Análise faunística de coleópteros coletados em plantas de Eucalyptus urophylla S.T. Blake e Eucalyptus saligna Sm. Piracicaba: Escola Superior de Agricultura "Luiz de Queiroz", 1984. 105 p. Dissertação (Mestrado em Entomologia) - Universidade de São Paulo, 1984.

CARVALHO, A. G.; FIGUEIRA, L. K. Biologia de Pygiopachimerus lineola (Chevrolat, 1871) (Coleoptera: Bruchidae) em frutos de Cassia javanica L. (Leguminosae Caesalpinioideae). Floresta e Ambiente, v. 6, n. 1, p. 8387, 1999.

CLARKE, A. R. et al. Local dispersion of the Eucalyptus leaf-beetle Chrysophtarta bimaculata (Coleoptera: Chrysomelidae), and implications for forest protection. Journal of Applied Ecology, v. 34, n. 3, p. 807-816, 1997.

FERRAZ, F. C. et al. Eficiência de armadilhas etanólicas para levantamentos de coleópteros do reflorestamento de Eucalyptus citriodora em Pinheiral, RJ. Floresta e Ambiente, v. 6, n. 1, p. 159-162, 1999.

FERREIRA, P. S. F; MARTINS, D. S. Contribuição ao método de captura de insetos por meio de armadilha luminosa, para a obtenção de exemplares sem danos morfológicos. Revista Ceres, v. 29, n. 165, p. 538-543, 1982.

GALLO, D. et al. Manual de entomologia agrícola. 2.ed. São Paulo: Agronômica Ceres, 1988. p. 649.

MEZZOMO, J. A. et al. Influência de faixas de vegetação nativa sobre Coleoptera em Eucalyptus cloeziana. Revista Árvore, v. 22, n. 1, p. 77-87, 1998.

MORALES, N. E. et al. Índices populacionais de besouros Scolytidae em reflorestamento de Eucalyptus grandis W. Hill ex. Maiden no município de Antônio Dias, Minas Gerais. Revista Árvore, v. 23, n. 3, p. 359-363, 1999. 
MORALES, N. E. et al. Fluctuación poblacional de Scolytidae (Coleoptera) en zonas reforestadas con Eucalyptus grandis (Myrtaceae) en Minas Gerais, Brasil.

Revista de Biologia Tropical, v. 48, n. 1, p. 101-107, 2000.

OHMART, C. P.; EDWARDS, P. B. Insect herbivory on Eucalyptus. Annual Review of Entomology, v. 36, p. 637657, 1991.

PEDROSA-MACEDO, J. H. Pragas Florestais do Sul do Brasil. Manual de Pragas em Florestas, v. 2, p. 112, 1993.

PEREIRA, L. G. B. et al. Percentual de mortalidade de lagartas de Thyrinteina arnobia (Stoll, 1782) (Lepidoptera: Geometridae) coletadas na bordadura e no interior de plantios de Eucalyptus grandis W. Hill ex Maiden.

Agrárias, v. 13, n. 1-2, p. 233-238, 1994.

PEREIRA, J. M. M. et al. Lepidoptera pests collected in Eucalyptus urophylla plantations during five years in Três Marias, State of Minas Gerais, Brazil. Revista de Biologia Tropical, v. 49, n. 3, 2001. (No prelo).

PINTO, R. et al. Flutuação populacional de Coleoptera em plantio de Eucalyptus urophylla no município de Três Marias, Minas Gerais. Floresta e Ambiente, v. 7, n. 1, p. 143-151, 2000 .

SANTOS, G. P.; ZANUNCIO, J. C.; ANJOS, N. Novos resultados sobre a biologia de Psorocampa denticulata Schaus (Lepidoptera: Notodontidae), desfolhadora de eucalipto. Revista Árvore, v. 6, n. 2, p. 121-132, 1982.

SANTOS, G. P. et al. Danos causados por Sennius cupreatus e S. spodiogaster (Coleoptera: Bruchidae) em sementes de Melanoxylon brauna. Revista Ceres, v. 38, n. 218, p. 315-322, 1991.

SANTOS, G. P. et al. Daños por Sennius amazonicus, Sennius sp. y Amblycerus sp. (Coleoptera: Bruchidae) en semillas de Sclerobium sp. (Leguminosae). Revista de Biologia Tropical, v. 45, n. 2, p. 883-886, 1997.
ZANUNCIO, J. C. et al. Aspectos biológicos de Blera varana (Lepidoptera: Notodontidae) desfolhadora de eucalipto. Anais da Sociedade Entomológica do Brasil, v. 20, n. 1, p. 35-43, 1991.

ZANUNCIO, J. C. et al. Principais lepidópteros, pragas primárias e secundárias, de Eucalyptus grandis na região de Guanhães, Minas Gerais, durante o período de junho de 1989 a maio de 1990. Científica, v. 20, n. 1, p. 145-155, 1992a.

ZANUNCIO, J. C. et al. Ciclo de vida e consumo foliar de Sarsina violascens (Herrich - Schaeffer, 1856)

(Lepidoptera, Lymantriidae), em Eucalyptus urophylla.

Revista Brasileira de Entomologia, v. 36, n. 4, p. 843-850, 1992b.

ZANUNCIO, J. C. et al. Coleópteros associados à eucaliptocultura nas regiões de São Mateus e Aracruz, Espírito Santo. Revista Ceres, v. 41, n. 232, p. 584-590. 1993a.

ZANUNCIO, J. C. et al. Levantamento e flutuação populacional de lepidópteros associados à eucaliptocultura: VI. Região de Belo Oriente, Minas Gerais. Pesquisa Agropecuária Brasileira, v. 28, n. 10, p. 1121-1127, 1993 b.

ZANUNCIO, J. C. et al. Major lepidopterous defoliators of eucalypt in southeast Brazil. Forest Ecology and Management, v. 65, p. 53-63, 1994a.

ZANUNCIO, T.V. et al. Biologia de Nystalea nyseus (Cramer, 1775) (Lepidoptera: Notodontidae) em folhas de Eucalyptus urophylla. Acta Amazônica, v. 34, n. 1/2, p. 153-160, 1994b.

ZANUNCIO, T.V. et al. Biologia de Euselasia hygenius (Lepidoptera, Riodinidae) e seu consumo foliar em Eucalyptus urophylla. Revista Brasileira de Entomologia, v. 39, n. 3, p. 487-492, 1995. 